\title{
Instability of a quantum spin liquid in an organic triangular-lattice antiferromagnet
}

\section{T. Itou ${ }^{1 \star}$, A. Oyamada', S. Maegawa' and R. Kato ${ }^{2}$}

Quantum liquids-known to be realized in ${ }^{3} \mathrm{He},{ }^{4} \mathrm{He}$ and electrons in metals-generally exhibit instabilities unforeseen under classical Newtonian dynamics, such as the superfluid and superconducting transitions. Recently, a new quantum liquid, now known as the quantum spin liquid, has been discovered in frustrated antiferromagnetic spin-1/2 systems ${ }^{1,2}$. In this state, quantum fluctuations of spins prevent classical antiferromagnetic ordering even at absolute zero, similar to the situation in the well-known quantum liquids. A fundamental question that has remained open is whether instabilities other than classical ordering can occur in a quantum spin liquid, as well as in the well-known quantum liquids. Here we demonstrate experimentally that a quantum spin liquid in an organic triangular-lattice antiferromagnet undergoes an instability involving symmetry breaking and/or topological ordering $^{3}$, possibly giving rise to a new quantum state of matter. Our result reveals a new variety of quantum-liquid instability, which might become a comparable concept to the already-known fermion-liquid instabilities (such as BardeenCooper-Schrieffer pairing and Peierls instability) and bosonliquid instability (Bose-Einstein condensation).

One of the most striking features deriving from quantum mechanics is the destabilization effect of classical ordering. In classical Newtonian dynamics, all systems lose their internal degrees of freedom and crystallize as the temperature tends towards zero. Quantum fluctuations work against this tendency and sometimes cause a quantum-liquid state without classical crystallization even at absolute zero. The best-known examples are ${ }^{3} \mathrm{He},{ }^{4} \mathrm{He}$ and electron systems in metals. Over the past one hundred years, these states have been intensively studied and shown to have several instabilities that are not identified with classical crystallization but described by quantum mechanics. One example is the Peierls instability in quantum fermion liquids, which breaks translational symmetry. Another profound example are the superfluid and superconducting instabilities, which have been one of the central topics in modern physics. Such instabilities arise because there is always an energy gain if a gapless quantum-liquid system creates a gap by undergoing symmetry breaking.

Recently, a new quantum liquid has been discovered ${ }^{1,2}$ - the quantum spin liquid. A classical spin system with antiferromagnetic interactions inevitably undergoes classical crystallization, that is, antiferromagnetic ordering. In spin-1/2 systems, quantum fluctuations take effect and destabilize the classical crystallization. If they completely destroy the classical crystallization, they may realize a quantum state with no trivial order, which is referred to as a quantum spin liquid. (Note that, in this definition, the quantum spin liquids may yet exhibit non-trivial symmetry breaking and/or topological ordering.) Apart from in one-dimensional (1D) systems, however, quantum fluctuations are so weak on typical non-frustrated lattices that they cannot destroy classical crystallization $^{4-6}$. Therefore, strong geometrical frustration, which works against the classical crystallization, is needed to realize the spin-liquid state for $D \geq 2$. This spin liquid, although theoretically proposed early on ${ }^{7}$, has not been experimentally realized for many years, because real model materials of ideal frustrated 1/2-spins are limited. Recently, however, experimental studies of spin-liquid physics have taken an important step; some organics are now recognized as ideal frustrated spin-1/2 systems, and the spin-liquid state has been found ${ }^{1,2}$ in $\kappa$ - $(\mathrm{BEDT}-\mathrm{TTF})_{2} \mathrm{Cu}_{2}(\mathrm{CN})_{3}(\mathrm{BEDT}-\mathrm{TTF}=$ bis(ethylenedithio)-tetrathiafulvalene) and $\mathrm{EtMe}_{3} \mathrm{Sb}\left[\mathrm{Pd}(\mathrm{dmit})_{2}\right]_{2}$ (dmit = 1,3-dithiole-2-thione-4,5-dithiolate, $\mathrm{Me}=\mathrm{CH}_{3}, \mathrm{Et}=$ $\mathrm{C}_{2} \mathrm{H}_{5}$ ), which have sparked considerable interest in the condensedmatter community ${ }^{8-25}$.

Thus, the first experimental goal of realizing the spin liquid in real materials has been achieved. The next stage is to address the fundamental question of whether the quantum spin liquid undergoes instabilities other than classical ordering, as in the typical well-known quantum liquids. Indeed, theorists have proposed several possible instabilities, such as spinon pairing ${ }^{15,16,25}$ and chiral ordering (chiral spin liquid) ${ }^{26,27}$. In the most-studied organic spinliquid material $\kappa$-(BEDT-TTF $)_{2} \mathrm{Cu}_{2}(\mathrm{CN})_{3}$, however, experimental reports on its low-temperature nature are controversial. Specificheat measurements suggest that this material exhibits a gapless state with a spinon Fermi surface ${ }^{28}$, whereas a thermal-conductivity study suggests a full gap at low temperatures ${ }^{29}$. Nuclear magnetic resonance (NMR) measurements show that the temperature dependence of the spin-lattice relaxation rates does not exhibit an obvious kink that clearly demonstrates a phase transition ${ }^{1,30}$. It is also noted that the spin-lattice relaxation functions become non-single exponential at low temperatures ${ }^{30}$, making it difficult to discuss the intrinsic spin dynamics and the gap structure of the spin excitation. Therefore, it is unresolved whether this spin liquid undergoes an instability involving the creation of a spin gap.

In this Letter, we report the discovery of spin-liquid instability found in a further spin-liquid material, EtMe $3 \mathrm{Sb}\left[\mathrm{Pd}(\mathrm{dmit})_{2}\right]_{2}$, which is a $2 \mathrm{D}$ triangular-lattice spin system with antiferromagnetic interactions $J=220-250 \mathrm{~K}$, as shown in Fig. 1. It has previously been found that this spin system maintains a gapless spinliquid state down to at least $1.37 \mathrm{~K}$ (ref. 2). We carried out ${ }^{13} \mathrm{C}$-NMR measurements under an applied field of $7.65 \mathrm{~T}$ at ultralow temperatures using a dilution refrigerator (see the Methods section), and found an instability in the spin-liquid state under the field, which involves non-trivial symmetry breaking and/or topological ordering.

Figure 2 shows the temperature dependence of the spin-lattice relaxation rate $\left(T_{1}^{-1}\right)$. We have fitted the relaxations of the nuclear magnetization $M(t)$ using the stretched-exponential function $1-M(t) / M(\infty)=\exp \left\{-\left(t / T_{1}\right)^{\beta}\right\}$, where $\beta$ (shown in Fig. 3)

${ }^{1}$ Graduate School of Human and Environmental Studies, Kyoto University, Kyoto 606-8501, Japan, ${ }^{2}$ Condensed Molecular Materials Laboratory, RIKEN, Wako-shi, Saitama 351-0198, Japan. *e-mail: tetsuaki@staff.mbox.media.kyoto-u.ac.jp. 

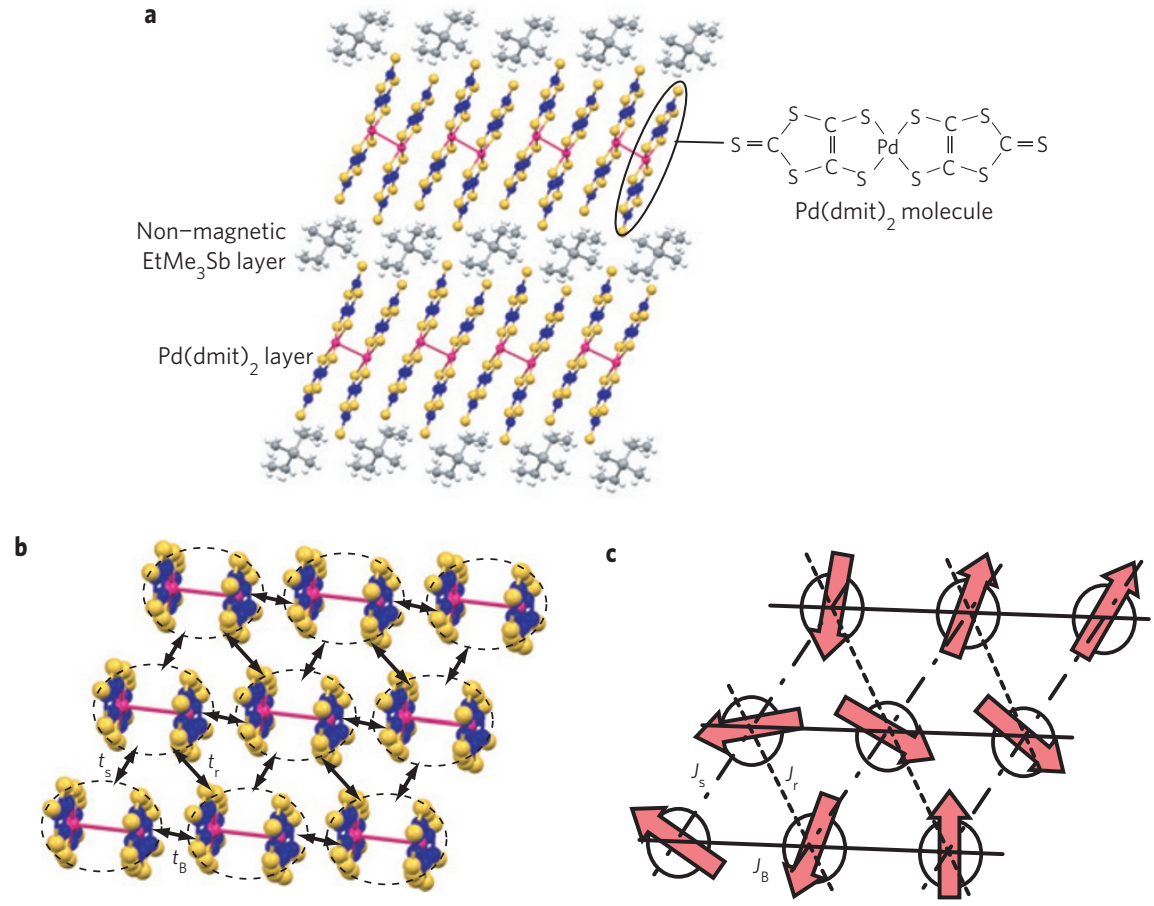

Figure 1 | Crystal structure of EtMe $\left.\mathrm{Sb}_{3} \mathrm{Sd}(\mathrm{Pdmit})_{2}\right]_{2}$. a, Side view of the crystal structure of $\mathrm{EtMe}_{3} \mathrm{Sb}\left[\mathrm{Pd}(\mathrm{dmit})_{2}\right]_{2}$. $2 \mathrm{D}$ magnetic $\mathrm{Pd}(\mathrm{dmit})_{2}$ layers are separated by non-magnetic layers of the closed-shell monovalent cation $\mathrm{EtMe}_{3} \mathrm{Sb}$. $\mathbf{b}$, Top view of the crystal structure of the Pd(dmit) 2 layer. The $\mathrm{Pd}(\mathrm{dmit})_{2}$ molecules are strongly dimerized (the pairs are denoted by dashed ovals). One electron with a $1 / 2$-spin is localized on each $\left[\mathrm{Pd}(\mathrm{dmit})_{2}\right]_{2}$ dimer. The arrows $\left(t_{B}, t_{s}\right.$ and $\left.t_{r}\right)$ indicate the transfer-integral network between the molecular orbitals of the $\left[\mathrm{Pd}(\mathrm{dmit})_{2}\right]_{2}$ dimers. The three transfer integrals are non-equivalent but close to each other ${ }^{2}$. c, Localized spin model applicable to EtMe $3 \mathrm{Sb}\left[\mathrm{Pd}(\mathrm{dmit})_{2}\right]_{2}$. Three exchange interactions $\left(J_{B}, J_{\mathrm{s}}\right.$ and $\left.J_{\mathrm{r}}\right)$ are close to each other, reflecting the values of $t_{\mathrm{B}}, t_{\mathrm{s}}$ and $t_{\mathrm{r}}$. The exchange interactions are estimated to be $220-250 \mathrm{~K}$ (ref. 2).

is the stretching exponent. The decrease in $\beta$ means that the relaxation function becomes non-single-exponential, indicating the distribution of the relaxation. This distribution increases from about $20 \mathrm{~K}$ and reaches a maximum around $1 \mathrm{~K}$ on cooling. In Fig. 2, we also show the relaxation rate determined from the initial decay slope of the relaxation curve. The difference between the two relaxation rates shows the degree of the distribution.

In spite of the distribution, it is clear from Fig. 2 that there is an obvious kink in the temperature dependence of $T_{1}^{-1}$ at around $1.0 \mathrm{~K}$. This strongly suggests that a phase transition occurs at this temperature. As no discontinuous jump is observed in the temperature dependence of $T_{1}^{-1}$, this is not a first-order but a continuous transition. The important point to note here is that continuous phase transitions always involve essential changes of states, that is, symmetry breaking and/or topological ordering. Therefore, our result indicates that the gapless spin liquid changes to an essentially different spin state with symmetry breaking and/or topological ordering.

This instability is not considered to be classical magnetic ordering, because the anomaly in $T_{1}^{-1}$ is not a critical divergence. Spectral analysis directly proves this point. Figure 4 shows the NMR spectra down to $19.4 \mathrm{mK}$. The transition around $1.0 \mathrm{~K}$ does not affect the spectral shape; all of the spectra are largely the same over the whole temperature region and the spectral tails are at most within $\pm 50 \mathrm{kHz}$. This width is much smaller than the scale of the hyperfine coupling constant of the ${ }^{13} \mathrm{C}$ sites, which is about $9 \times 10^{2} \mathrm{kHz} / \mu_{\mathrm{B}}$ (ref. 2), where $\mu_{\mathrm{B}}$ is the Bohr magneton. Therefore, classical spin ordering and freezing are clearly absent down to $19.4 \mathrm{mK}$, which proves that the present instability differs from classical ordering/freezing.

The high-temperature phase above the transition does not have a spin gap, because $T_{1}^{-1}$ does not vanish in the low-temperature limit. In contrast, the low-temperature phase below the transition

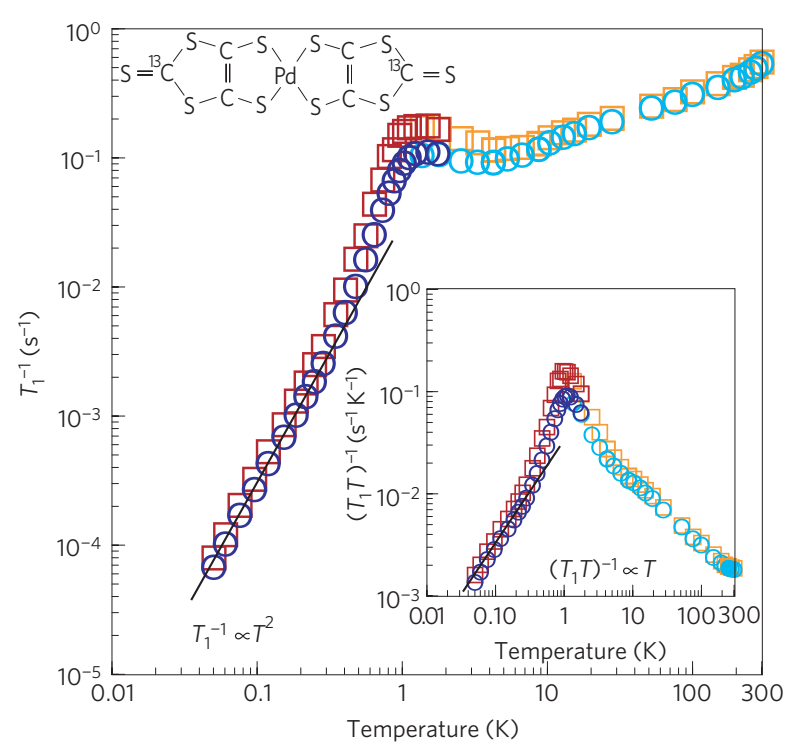

Figure 2 | Temperature dependence of the ${ }^{13} \mathrm{C}$ nuclear spin-lattice relaxation rate of $\mathrm{EtMe}_{3} \mathrm{Sb}\left[\mathrm{Pd}(\mathrm{dmit})_{2}\right]_{2}$. The main graph shows the ${ }^{13} \mathrm{C}$ nuclear spin-lattice relaxation rate $T_{1}^{-1}$ of $\mathrm{EtMe}_{3} \mathrm{Sb}\left[\mathrm{Pd}(\mathrm{dmit})_{2}\right]_{2}$, and the inset graph shows $\left(T_{1} T\right)^{-1}$, where $T$ is temperature. The circles indicate the values determined from the stretched-exponential analysis (see text), and the squares denote the values determined from the initial decay slopes of the relaxation curves. The dark blue circles and dark red squares are obtained from the present measurements below $1.75 \mathrm{~K}$ in a dilution refrigerator. For clarity, we did the same analysis for previously reported ${ }^{2}$ higher-temperature data above $1.37 \mathrm{~K}$, and show them here as light blue circles and light red squares. Inset: $\mathrm{Pd}(\mathrm{dmit})_{2}$ molecule with selective substitution of the ${ }^{13} \mathrm{C}$ isotope for the present ${ }^{13} \mathrm{C}$-NMR measurement. 

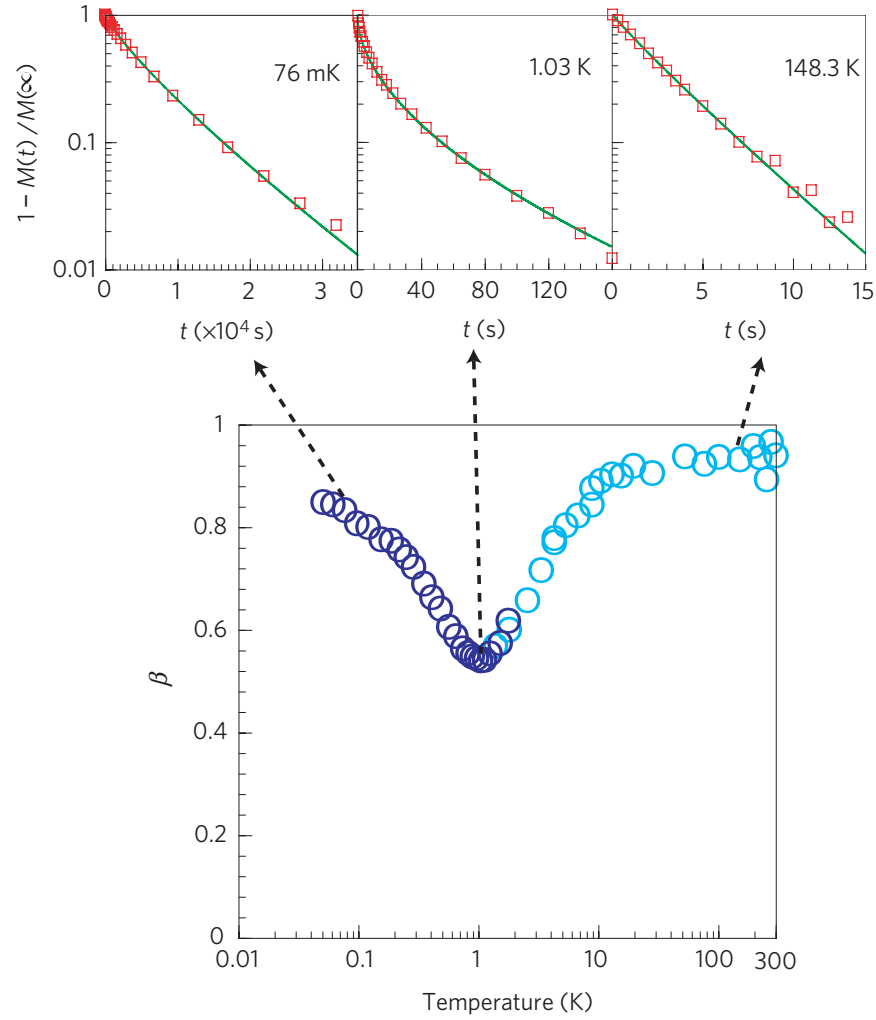

Figure 3 | Stretching exponent obtained from the ${ }^{13} \mathrm{C}$ nuclear spin-lattice relaxation curves. The main panel shows the temperature dependence of the exponent. The dark blue circles show values obtained from the present measurements in a dilution refrigerator. We also show reanalysed values for previously reported ${ }^{2}$ higher-temperature data as light blue circles. The spin-lattice relaxation curves at three representative temperatures are presented in the upper three panels, where the red squares indicate obtained experimental data and the green lines represent fits to stretched-exponential functions.

shows a steep decrease of $T_{1}^{-1}$ on cooling. At sufficiently low temperatures, the spin-lattice relaxation curves recover to singleexponential functions as shown in Fig. 3. This is different from the case of $\kappa$-(BEDT-TTF $)_{2} \mathrm{Cu}_{2}(\mathrm{CN})_{3}$ at low temperatures, where the relaxation curves become further from single exponential functions with decreasing temperature ${ }^{30}$, and makes it difficult to discuss the intrinsic spin dynamics. In the low-temperature region of $\mathrm{EtMe}_{3} \mathrm{Sb}\left[\mathrm{Pd}(\mathrm{dmit})_{2}\right]_{2}$ where the relaxation curves recover to single-exponential functions, we can see from Fig. 2 that $T_{1}^{-1}$ is proportional to the square of the temperature. This means that the imaginary part of the $q$-integrated dynamic susceptibility (to be exact, $\left.\lim _{\omega \rightarrow 0} \Sigma_{q} \chi^{\prime \prime}(q, \omega) / \omega\right)$, which is evaluated from $\left(T_{1} T\right)^{-1}$, decreases in proportion to the temperature on cooling, as shown in the inset of Fig. 2 ( $q$ : wave vector, $\omega$ : frequency). This is in contrast to the nature of the fully gapless spin liquid with a spinon Fermi surface, where the imaginary part of the susceptibility remains constant (Fermi-liquid case) or diverges (non-Fermi-liquid case) on cooling. Thus, the low-temperature phase is not fully gapless, and therefore has a spin gap at least in some portion of $q$-space.

We emphasize that the decrease in the imaginary part of the susceptibility does not follow an exponential law but a power law in temperature. This result implies that the spin gap may be a nodal one, similar to superconducting gaps in anisotropic superconductors, often realized in correlated quantum fermion liquids. Although it might also be possible that the system has a full gap and that $T_{1}^{-1}$ at low temperatures reflects extrinsic relaxation, this is more unlikely. In this case, the relaxation curves would

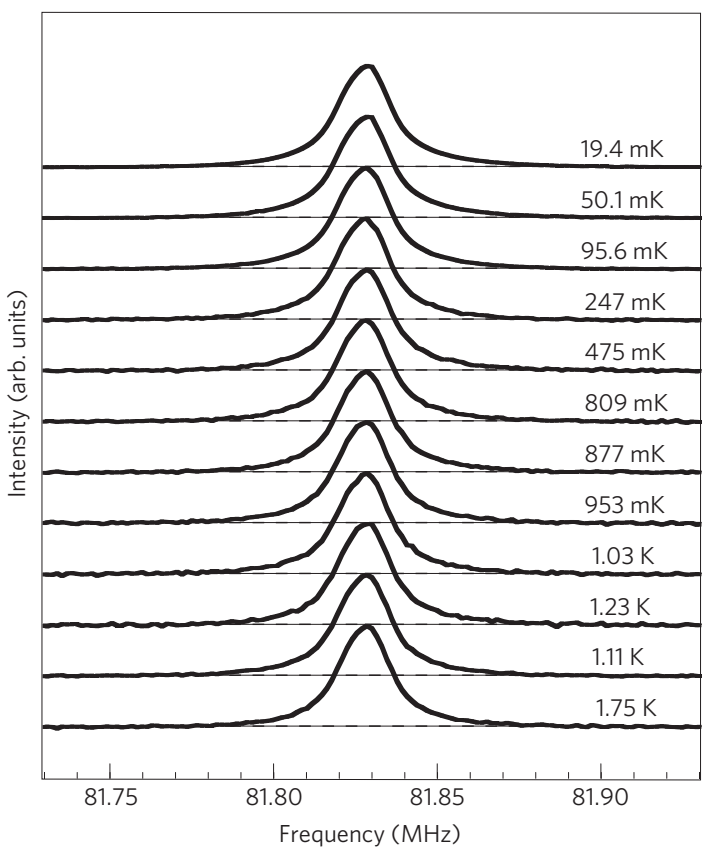

Figure $4 \mid{ }^{13} \mathrm{C}$-NMR spectra of $\mathrm{EtMe}_{3} \mathrm{Sb}\left[\mathrm{Pd}(\mathrm{dmit})_{2}\right]_{2}$ at several ultralow temperatures measured in a dilution refrigerator. The spectra are obtained by Fourier transformation of the spin-echo signals for randomly oriented single crystals.

become more or less distributed non-single-exponential functions. Experimental results instead show that the relaxation curves recover to a single-exponential function in the low-temperature limit, as shown in Fig. 3. Therefore, it is more likely that the $T^{2}$ dependence of $T_{1}^{-1}$ is intrinsic and that the spin gap is nodal.

In principle, this spin gap should be observable also in the behaviour of the static spin susceptibility. However, the susceptibility was so far measured only down to $5 \mathrm{~K}$ and is not available in the region below the transition temperature ${ }^{2}$. We also note that it will be difficult to measure the intrinsic susceptibility below the transition temperature, because the Curie term caused by impurity free spins will make a serious contribution at such low temperatures even for a very small number of impurities. The Knight shift (the first moment of the spectrum) offers another way to measure the static spin susceptibility. It is expected that the spin gap leads to the disappearance of the spin susceptibility, yielding the disappearance of the Knight shift of a few kilohertz through the hyperfine coupling of about $9 \times 10^{2} \mathrm{kHz} / \mu_{\mathrm{B}}$ (ref. 2). Unfortunately, our experimental results do not have the accuracy to discuss such a small shift because of the comparatively large spectral width and the slight extrinsic drift of the external applied field, which is inevitable even when using a superconducting magnet with high stability (see the Methods section).

In summary, our NMR experiments show that the spin system of $\mathrm{EtMe}_{3} \mathrm{Sb}\left[\mathrm{Pd}(\mathrm{dmit})_{2}\right]_{2}$ does not undergo classical ordering/freezing down to $19.4 \mathrm{mK}$, which is less than $0.01 \%$ of $J$. Whereas this quantum spin liquid has a gapless spin excitation above $1.0 \mathrm{~K}$, we found clear evidence that the spin system under $7.65 \mathrm{~T}$ shows an instability other than classical ordering at around $1.0 \mathrm{~K}$ and acquires a spin gap. This gap may be nodal, similar to that of anisotropic superconductivity.

Last, we mention future debatable problems on the instability that we have discovered. One of the possible candidates is the pairing instability of the spinon Fermi surface. This naturally explains the nodal-gap formation when spinon pairing occurs in a non-s-wave Bardeen-Cooper-Schrieffer channel and causes an anisotropic (such as $d$-wave) resonating-valence-bond state. 
Such a state is expected to have the same nodal gap as that of a corresponding superconducting state, and thus, may explain the gap structure in the present low-temperature phase. We should point out, however, that $T_{1}^{-1}$ in the present system is almost constant just above the transition temperature and has $T^{2}$ dependence in the low-temperature limit. This behaviour does not completely correspond to that of a 2D anisotropic superconductor with line nodes, in which the constant and $T^{2}$ behaviour is observed not in $T_{1}^{-1}$ but in $\left(T_{1} T\right)^{-1}$. The present unprecedented excitation will be a key factor in understanding the new type of quantum liquid and instability realized in a quantum-spin system on a triangular lattice.

\section{Methods}

We prepared fine high-quality single crystals of $\mathrm{EtMe}_{3} \mathrm{Sb}\left[\mathrm{Pd}(\mathrm{dmit})_{2}\right]_{2}$ with typical sizes of $0.3 \mathrm{~mm} \times 0.3 \mathrm{~mm} \times 0.01 \mathrm{~mm}$, using an aerial oxidation method. For the ${ }^{13} \mathrm{C}$-NMR measurements, the carbon atoms at both ends of the $\mathrm{Pd}(\mathrm{dmit})_{2}$ molecules were enriched with ${ }^{13} \mathrm{C}$ isotopes, as shown in the inset of Fig. 2. The NMR measurements were carried out at a field of $7.65 \mathrm{~T}$ using many single crystals with no particular orientation packed into a glass tube. The field was applied by a superconducting magnet with very high stability, which restrains the drift of the applied field within about 1-2 gauss (corresponding to the ${ }^{13} \mathrm{C}-\mathrm{NMR}$ frequency of $1-2 \mathrm{kHz}$ ). The NMR spectra were obtained by Fourier transformation of the spin-echo signals following a $\pi / 2-\pi$ pulse sequence. The typical pulse widths of $\pi / 2$ and $\pi$ were 3 and $6 \mu$ s, respectively. These values were sufficiently smaller than the inverse of the spectral widths, and thus the pulses could cover the whole NMR spectra. The spin-lattice relaxation curves were obtained from the integrated spin-echo intensity $M(t)$ after time delay $t$ following saturation comb pulses.

Received 10 December 2009; accepted 28 May 2010; published online 11 July 2010

\section{References}

1. Shimizu, Y., Miyagawa, K., Kanoda, K., Maesato, M. \& Saito, G. Spin liquid state in an organic Mott insulator with a triangular lattice. Phys. Rev. Lett. 91, 107001 (2003)

2. Itou, T., Oyamada, A., Maegawa, S., Tamura, M. \& Kato, R. Quantum spin liquid in the spin-1/2 triangular antiferromagnet $\mathrm{EtMe}_{3} \mathrm{Sb}\left[\mathrm{Pd}(\mathrm{dmit})_{2}\right]_{2}$. Phys. Rev. B 77, 104413 (2008)

3. Wen, X-G. Topological orders in rigid states. Int. J. Mod. Phys. B 4, 239-271 (1990).

4. Dyson, F. J., Lieb, E. H. \& Simon, B. Phase-transitions in quantum spin systems with isotropic and non-isotropic interactions. J. Stat. Phys. 18, 335-383 (1978).

5. Kennedy, T., Lieb, E. H. \& Shastry, B. S. Existence of Néel order in some spin-1/2 Heisenberg antiferromagnets. J. Stat. Phys. 53, 1019-1030 (1988).

6. Reger, J. D. \& Young, A. P. Monte Carlo simulations of the spin-1/2 Heisenberg antiferromagnet on a square lattice. Phys. Rev. B 37, 5978-5981 (1988).

7. Anderson, P. W. Resonating valence bonds: A new kind of insulator? Mater. Res. Bull. 8, 153-160 (1973).

8. Lee, S-S. \& Lee, P.A. U(1) gauge theory of the Hubbard model: Spin liquid states and possible application to $\kappa$-(BEDTTTF $)_{2} \mathrm{Cu}_{2}(\mathrm{CN})_{3}$. Phys. Rev. Lett. 95, 036403 (2005)

9. Motrunich, O. I. Variational study of triangular lattice spin-1/2 model with ring exchanges and spin liquid state in $\kappa-(\mathrm{ET})_{2} \mathrm{Cu}_{2} \mathrm{CN}_{3}$. Phys. Rev. B 72, 045105 (2005).

10. Kyung, B. \& Tremblay, A-M. S. Mott transition, antiferromagnetism, and $d$-wave superconductivity in two-dimensional organic conductors. Phys. Rev. Lett. 97, 046402 (2006).

11. Powell, B. J. \& McKenzie, R. H. Strong electronic correlations in superconducting organic charge transfer salts. J. Phys. Condens. Matter 18, R827-R866 (2006).
12. Mizusaki, T. \& Imada, M. Gapless quantum spin liquid, stripe, and antiferromagnetic phases in frustrated Hubbard models in two dimensions. Phys. Rev. B 74, 014421 (2006).

13. Yunoki, S. \& Sorella, S. Two spin liquid phases in the spatially anisotropic triangular Heisenberg model. Phys. Rev. B 74, 014408 (2006).

14. Motrunich, O. I. Orbital magnetic field effects in spin liquid with spinon Ferm sea: Possible application to $\kappa-(\mathrm{ET})_{2} \mathrm{Cu}_{2} \mathrm{CN}_{3}$. Phys. Rev. B 73, 155115 (2006).

15. Lee, S-S., Lee, P. A. \& Senthil, T. Amperean pairing instability in the U(1) spin liquid state with Fermi surface and application to $\kappa$-(BEDTTTF $)_{2} \mathrm{Cu}_{2}(\mathrm{CN})_{3}$. Phys. Rev. Lett. 98, 067006 (2007).

16. Galitski, V. \& Kim, Y. B. Spin-triplet pairing instability of the spinon Fermi surface in a U(1) spin liquid. Phys. Rev. Lett. 99, 266403 (2007).

17. Senthil, T. Theory of a continuous Mott transition in two dimensions. Phys. Rev. B 78, 045109 (2008).

18. Qi, Y. \& Sachdev, S. Insulator-metal transition on the triangular lattice. Phys. Rev. B 77, 165112 (2008).

19. Yoshioka, T., Koga, A. \& Kawakami, N. Quantum phase transitions in the Hubbard model on a triangular lattice. Phys. Rev. Lett. 103, 036401 (2009).

20. Tocchio, L. F., Parola, A., Gros, C. \& Becca, F. Spin-liquid and magnetic phases in the anisotropic triangular lattice: The case of $\kappa-(\mathrm{ET})_{2} \mathrm{X}$. Phys. Rev. B 80, 064419 (2009).

21. Heidarian, D., Sorella, S. \& Becca, F. Spin-1/2 Heisenberg model on the anisotropic triangular lattice: From magnetism to a one-dimensional spin liquid. Phys. Rev. B 80, 012404 (2009).

22. Qi, Y., Xu, C. \& Sachdev, S. Dynamics and transport of the $\mathrm{Z}_{2}$ spin liquid: Application to $\kappa-(\mathrm{ET})_{2} \mathrm{Cu}_{2} \mathrm{CN}_{3}$. Phys. Rev. Lett. 102, 176401 (2009).

23. Xu, C. \& Sachdev, S. Global phase diagrams of frustrated quantum antiferromagnets in two dimensions: Doubled Chern-Simons theory. Phys. Rev. B 79, 064405 (2009).

24. Gregor, K. \& Motrunich, O. I. Nonmagnetic impurities in a $S=1 / 2$ frustrated triangular antiferromagnet: Broadening of ${ }^{13} \mathrm{C}$ NMR lines in $\kappa-(\mathrm{ET})_{2} \mathrm{Cu}_{2} \mathrm{CN}_{3}$. Phys. Rev. B 79, 024421 (2009).

25. Grover, T., Trivedi, N., Senthil, T. \& Lee, P. A. Weak Mott insulators on the triangular lattice: possibility of a gapless nematic quantum spin liquid. Phys. Rev. B 81, 245121 (2010).

26. Kalmeyer, V. \& Laughlin, R. B. Equivalence of the resonating-valence bond and fractional quantum Hall states. Phys. Rev. Lett. 59, 2095-2098 (1987).

27. Baskaran, G. Novel local symmetries and chiral-symmetry-broken phases in $\mathrm{S}=1 / 2$ triangular-lattice Heisenberg model. Phys. Rev. Lett. 63, 2524-2527 (1989).

28. Yamashita, S. et al. Thermodynamic properties of a spin-1/2 spin-liquid state in a $\kappa$-type organic salt. Nature Phys. 4, 459-462 (2008).

29. Yamashita, M. et al. Thermal-transport measurements in a quantum spin-liquid state of the frustrated triangular magnet $\kappa$-(BEDT-TTF $)_{2} \mathrm{Cu}_{2}(\mathrm{CN})_{3}$. Nature Phys. 5, 44-47 (2009).

30. Shimizu, Y., Miyagawa, K., Kanoda, K., Maesato, M. \& Saito, G. Emergence of inhomogeneous moments from spin liquid in the triangular-lattice Mott insulator $\kappa-(\mathrm{ET})_{2} \mathrm{Cu}_{2} \mathrm{CN}_{3}$. Phys. Rev. B 73, 140407 (2006).

\section{Acknowledgements}

We acknowledge helpful discussions with K. Kanoda, M. Tamura and Y. Shimizu. We thank F. Kagawa for helpful suggestions for improving of the manuscript. This work was supported by Grant-In-Aid for Scientific Research from MEXT, Japan (numbers 16GS0219, 18740199, 19052005 and 21740255).

\section{Author contributions}

T.I. designed the experiments, and carried out the NMR measurements, data analysis and discussion; A.O. and S.M. provided experimental support and suggestions; R.K prepared the single crystals of $\mathrm{EtMe}_{3} \mathrm{Sb}\left[\mathrm{Pd}(\mathrm{dmit})_{2}\right]_{2}$ with selective substitution of the ${ }^{13} \mathrm{C}$ isotope and gave several suggestions; T.I. wrote, and all authors commented on, the manuscript.

\section{Additional information}

The authors declare no competing financial interests. Reprints and permissions information is available online at http://npg.nature.com/reprintsandpermissions. Correspondence and requests for materials should be addressed to T.I. 